\title{
artigo
}

\section{Educação em saúde na pandemia: atividades realizadas pela residência multiprofissional na vacinação contra influenza}

\author{
Health education in pandemia: activities carried out by the multiprofessional residence in vaccination against influenza \\ La educación sanitaria en la pandemia: actividades realizadas por la residencia multiprofesional en vacunación contra \\ la influenza
}

\begin{abstract}
RESUMO
O presente estudo tem como objetivo relatar experiências vivenciadas pela Equipe de Residentes Multiprofissionais em uma Unidade Básica de Saúde de Fortaleza, Ceará, Brasil, sobre as ações de educação em saúde realizadas junto à Campanha de Vacinação contra a Influenza durante a pandemia da Covid-19 no ano de 2020, que precisou passar por uma série de adaptações considerando esse novo contexto. Utilizou-se como metodologia um relato de experiência, de caráter descritivo e de natureza qualitativa. A fim de conseguir vacinar um grande número de pessoas evitando-se ajuntamentos, mostrou-se necessário realizar várias atividades de educação em saúde. A adoção das formas educativas relacionadas neste trabalho mostrou que apesar de um contexto novo de pandemia, as atividades realizadas uniram conhecimento técnico e prático em uma dada realidade de saúde, compartilhando-se informações importantes entre usuários, familiares e profissionais, o que refletiu no cuidado de saúde das pessoas e da comunidade.
\end{abstract}

DESCRITORES: Educação em Saúde; Equipe Multiprofissional; Campanha de Vacinação.

\section{ABSTRACT}

This study aims to report experiences lived by the Multiprofessional Residents Team in a Basic Health Unit in Fortaleza, Ceará, Brazil, about the health education actions carried out with the Influenza Vaccination Campaign during the Covid-19 pandemic, in the year 2020, which had to go through a series of adaptations considering this new context. An experience report, descriptive and qualitative in nature, was used as methodology. In order to be able to vaccinate a large number of people by avoiding gatherings, it was necessary to carry out various health education activities. The adoption of the educational forms listed in this work showed that despite a new pandemic context, the activities carried out brought together technical and practical knowledge in a given health reality, sharing important information between users, family members and professionals, which reflected in the care health of people and the community.

DESCRIPTORS: Health Education; Patient Care Team; Immunization Programs.

\section{RESUMEN}

El presente estudio tiene como objetivo reportar las experiencias vividas por el Equipo de Residentes Multiprofesionales en una Unidad Básica de Salud en Fortaleza, Ceará, Brasil, sobre las acciones de educación para la salud realizadas con la Campaña de Vacunación Influenza durante la pandemia de Covid-19 en el año 2020, que debió sufrir una serie de adaptaciones debido al contexto de la pandemia. Se utilizó como metodología una descripción de la experiencia, de carácter descriptivo y de carácter cualitativo. Para poder vacunar a un gran número de personas evitando las tertulias, fue necesario realizar diversas actividades de educación sanitaria. La adopción de las formas educativas enumeradas en este trabajo mostró que a pesar de un nuevo contexto pandémico, las actividades realizadas reunieron conocimientos técnicos y prácticos en una determinada realidad de salud, compartiendo información importante entre usuarios, familiares y profesionales, que se reflejó en la atención de la salud de las personas y la comunidad.

DESCRIPTORES: Educación en Salud; Grupo de Atención al Paciente; Programas de Inmunización.

RECEBIDO EM: 30/01/2021 APROVADO EM: 22/03/2021 


\section{Natiane do Nascimento Colares Bitu}

Cirurgiã-dentista. Residente R2 da Residência Multiprofissional - Residência Integrada em Saúde - da Escola de Saúde Pública do Ceará, Brasil (ESP-CE). Especialista em Saúde da Família pela Universidade da Integração Internacional da Lusofonia Afro-Brasileira (UNILAB).

ORCID: 0000-0003-3912-197X

\section{Roberta Pereira de Oliveira}

Assistente Social. Residente R2 da Residência Multiprofissional - Residência Integrada em Saúde - da Escola de Saúde Pública do Ceará, Brasil (ESP-CE). Especialista em Saúde da Família pela Universidade da Integração Internacional da Lusofonia Afro-Brasileira (UNILAB). Especialista em Políticas Públicas, Direitos Sociais e Serviço Social pela Universidade Estadual do Ceará (UECE). ORCID: 0000-0001-9534-7602

\section{Emmanuela Parente Rebouças Pinto}

Cirurgiã-dentista da Estratégia Saúde da Família de Fortaleza, Ceará, Brasil. Mestranda em Ensino na Saúde pela Universidade Estadual do Ceará (UECE).

ORCID: 0000-0002-1890-3596

\section{Antonio Rogério Mariz Santos}

Cirurgião-dentista da Estratégia Saúde da Família de Fortaleza, Ceará, Brasil. Especialista em Saúde da Família pela Universidade Federal do Ceará (UFC).

ORCID: 0000-0002-4790-1151

\section{INTRODUÇÃO}

A vacinação apresenta-se como uma prática muito importante de proteção imunológica dos seres humanos, sendo uma das principais ações de intervenção em saúde pública no controle de doenças provocadas por agentes imunizáveis, apresentando eficácia incontestável, já que através de sua utilização já foi alcançada a erradicação de doenças como poliomielite e varíola ${ }^{1,2}$.

O Programa Nacional de Imunização-PNI, criado em 1973, é coordenado pelo Ministério da Saúde de forma compartilhada com as Secretarias Estaduais e Municipais de Saúde, e tem como principal objetivo organizar a Política Nacional de Imunização, contribuindo para o controle, diminuição e/ou eliminação de doenças, como as ocasionadas pelo vírus da influenza, dentre outras ${ }^{1}$.

A influenza caracteriza-se como uma infecção viral aguda que afeta o sistema respiratório, causada pelos vírus $\mathrm{A}, \mathrm{B}, \mathrm{C}$ e $D$, apresentando tendência a se disseminar facilmente em epidemias sazonais, podendo também causar pandemias, pois é uma doença de elevada transmissibilidade e distribuição global ${ }^{3}$.

O PNI incorporou, em 1999, a estratégia de vacinação contra a influenza com o intuito de reduzir internações, complicações e mortes em população de alto risco. Em 2020 foi realizada a $22^{\circ}$ Campanha Nacional de Vacinação contra a Influenza, que estava prevista para ocorrer nos meses de março a maio de 2020, tendo como público alvo indivíduos com fatores ou condições de risco, acrescido de professores, puérperas, trabalhadores da saúde, adolescentes e jovens de 12 a 21 anos de idade sob medidas socioeducativas, população privada de liberdade, funcionários do sistema prisional e forças de segurança e salvamento, apresentando uma parcela da população estimada em 67,6 milhões de pessoas, com meta de vacinação de $90 \%$ do grupo elegível ${ }^{4}$.

De acordo com o Informe Técnico do Ministério da Saúde, que trata sobre a 22a Campanha Nacional de Vacinação contra a Influenza (2020), até o ano de 2019 a vacina estava disponível para adultos de 60 anos e mais idade, porém, em 2020, esse acesso foi ampliado para os grupos mais vulneráveis, incluindo também pessoas de 55 anos a 59 anos de idade e pessoas com deficiência ${ }^{5}$.

Para o ano de 2020, entretanto, a campanha de imunização precisou passar por uma série de adaptações devido ao contexto da pandemia ocasionada pelo novo Coronavírus SARS-CoV-2. Consoante infor- mações obtidas no Plano de Contingência Nacional para Infecção Humana pelo novo Coronavírus COVID-19, redigido pelo Ministério da Saúde em fevereiro de 2020, em 29 de dezembro de 2019, um hospital em Wuhan admitiu quatro pessoas com pneumonia e reconheceu que as quatro haviam trabalhado no Mercado Atacadista de Frutos do Mar de Huanan, lugar onde são vendidas aves vivas, produtos aquáticos e vários tipos de animais selvagens ao público. O hospital reportou essa ocorrência ao Centro de Controle de Doenças (CDC-China) e os epidemiologistas de campo da China (FETP-China) encontraram pacientes adicionais vinculados ao mercado e, em 30 de dezembro, as autoridades de saúde da província de Hubei notificaram esse cluster ao CDC da China ${ }^{3}$.

Com o objetivo de diminuir a transmissibilidade da Covid-19, retardando a epidemia no país, o Ministério da Saúde, lança, em março de 2020, uma Nota Informativa com recomendações sobre as medidas de saúde pública de prevenção à disseminação do novo Coronavírus, que trouxe estratégias a fim de evitar grandes aglomerações de pessoas durante a campanha de vacinação contra a influenza e contra o sarampo, também ${ }^{4}$.

Devido ao momento de pandemia, objetivou-se vacinar o maior número de 
pessoas, entre o público-alvo, mas optando por reorganizar o processo de trabalho, para evitar aglomerações. Nisso, foi orientado que as Secretarias Municipais de Saúde de cada município brasileiro estabelecessem parcerias com instituições públicas e privadas, a fim de descentralizar o máximo possível a vacinação para além das Unidades de Atenção Primária à Saúde, pois nesse local estaria concentrado um grande número de casos suspeitos de Covid- $19^{3}$. A fim de conseguir atingir esse objetivo, de vacinar um grande número de pessoas evitando-se ajuntamentos, mostrou-se necessário realizar atividades de educação em saúde.

A Educação em Saúde apresenta-se como um conjunto de ações que têm como objetivo promover a autonomia sobre o cuidado pessoal e coletivo no processo saúde-doença, sendo compreendida por três atores principais: a população em geral, os profissionais de saúde, e a gestão ${ }^{5}$.

No contexto de Pandemia da Covid-19, com um vírus novo que causa uma doença totalmente recente, sem evidências científicas de como tratá-la, e sem vacinação prévia, como a influenza, as atividades de educação em saúde fizeram-se bastante evidentes, uma vez que foi extremamente necessário educar a população sobre hábitos básicos de higiene, cuidados pessoais e coletivos, evitando aglomerações, além da necessidade de sensibilização sobre os cuidados e necessidade de manter a saúde estável e a vacinação de outras doenças atualizada.

Nesse aspecto, o presente estudo tem como objetivo relatar experiências vivenciadas pela Equipe de Residentes Multiprofissionais em uma Unidade Básica de Saúde de Fortaleza, Ceará, Brasil, sobre as ações de educação em saúde realizadas junto a Campanha de Vacinação contra a Influenza durante a pandemia da Covid-19.

\section{MÉTODO}

A metodologia deste relato foi baseada no tipo de pesquisa descritiva que, caracteriza-se como um estudo em que os pesquisadores, habitualmente, estão preocu- pados com a atuação prática, tendo como principal objetivo a descrição das características de determinada situação, população ou fenômeno ${ }^{6}$, enquadrando-se em um campo de pesquisa social que permite a obtenção de novos conhecimentos no campo da realidade e saber social ${ }^{7}$.

A pesquisa buscou descrever um relato de experiência, que caracteriza-se como a descriçãa de ações tomadas e impressões vivenciadas por pessoas em determinada situação ou momento, assim como aspectos que possam impactar de alguma forma no relato

A pesquisa buscou descrever um relato de experiência, que caracteriza-se como a descrição de ações tomadas e impressões vivenciadas por pessoas em determinada situação ou momento, assim como aspectos que possam impactar de alguma forma no relato ${ }^{8}$.

Com relação à natureza, trata-se de uma análise qualitativa, pois objetivou interpretar e analisar criticamente uma determinada situação e o impacto que traz na vida dos indivíduos envolvidos ${ }^{6}$.
$\mathrm{O}$ presente estudo traz um relato de experiência da intervenção de uma Equipe de Residentes Multiprofissionais durante a Campanha de Vacinação da Influenza, frente à pandemia da Covid-19 em uma Unidade de Atenção Primária à Saúde-UAPS de Fortaleza-CE. A integração da equipe multidisciplinar na campanha ocorreu nos meses de março a junho de 2020 , com abordagem de todos os públicos previstos durante a campanha.

Fizeram parte do público de vacinação contra a Influenza, assim como foram incluídas nesse estudo, pessoas com mais de 60 anos, trabalhadores da área da saúde, profissionais das forças de segurança e salvamento, pacientes com doenças crônicas (hipertensão, diabetes, asma), caminhoneiros, crianças de 6 meses a menores de 6 anos incompletos, professores, pessoas entre 55 e 60 anos incompletos, gestantes e puérperas. Todo esse público foi dividido de acordo com o Calendário Nacional de Vacinação, obedecendo uma cronologia de prioridades de vacinação. Foram excluídos os usuários não cadastrados nas UAPS de Fortaleza-CE, e os que, por algum motivo, não apresentaram, no ato da vacinação, os documentos necessários.

Desse modo, em um primeiro momento, a equipe responsável pela imunização precisou organizar-se para a campanha contra influenza e montou um espaço estratégico na outra entrada da UAPS, de atendimento às pessoas não sintomáticas. Logo na porta, antes de os usuários adentrarem a Unidade, havia um momento de educação em saúde por meio da fala da equipe de Residência Integrada em Saúde informando sobre os grupos prioritários, quais documentos necessários para ter acesso à vacina e a importância da população usar a máscara e higienizar as mãos, ressaltando a importância do isolamento social naquele momento, além da organização de filas com o correto distanciamento entre as pessoas para a entrada individual de pacientes na sala de imunização.

Quando a pandemia aumentou ainda mais seus números de infectados, fez-se necessário que a equipe responsável pela imunização realizasse articulação com 
algum equipamento social do território de abrangência, para que o trabalho de imunização contra influenza continuasse, sendo acordada uma parceria com a igreja católica do bairro, localizada em frente à Unidade de Saúde. A atuação junto à comunidade não parou em nenhum momento e as atividades continuaram, dessa vez em ambiente aberto, ao ar livre, no pátio da igreja.

Para esse trabalho de imunização com educação em saúde, estiveram presentes uma enfermeira, duas técnicas em enfermagem e a equipe de residentes da Escola de Saúde Pública do Ceará, cuja composição era formada por Assistente Social, Cirurgiã-dentista, Enfermeiras, Fisioterapeuta e Psicóloga. Mesmo com categorias profissionais diferentes, todas as profissionais participaram de forma ativa, tendo conhecimento e entendimento de todos os processos, inclusive do manuseio no sistema.

Ao final do dia, todos se dirigiam à sala de vacina da UAPS e contabilizavam o que havia sido utilizado de material, além de avaliarem aquele dia de trabalho e se havia algo que precisasse ser modificado, apresentando qualquer sugestão de mudanças à gestora da Unidade de Saúde, sendo acordado o melhor fluxo, sempre seguindo as orientações da Coordenadoria Regional de Saúde da Secretaria Regional V da Prefeitura Municipal de Fortaleza, Ceará, que por sua vez, seguia as orientações das autoridades sanitárias consideradas de maior instância.

Após alguns dias de trabalho externo, a equipe de vacinação retornou novamente para a UAPS, dando continuidade à imunização contra influenza, juntamente com as outras vacinas do calendário de imunização já previstas e consideradas de rotina. $\mathrm{O}$ trabalho externo envolveu também imunização em domicílio das pessoas super idosas (acima de 80 anos) e das pessoas com dificuldade de locomoção da comunidade essa demanda era repassada pelos Agentes Comunitários de Saúde-ACS à coordenação da Unidade, que gerenciava o atendimento domiciliar quando necessário.

Após o desenvolvimento das ações de educação em saúde juntamente com o trabalho multiprofissional necessário para a vacinação de todos os grupos prioritários da Campanha de Vacina contra a Influen$\mathrm{za}$, os dados desse estudo foram analisados e tratados a partir do relato dos próprios usuários, da gestora da UAPS e dos profissionais da Atenção Primária pertencente a unidade, assim como da observância da melhoria de organização e diminuição do contágio pelo Novo Coronavírus durante a Campanha.

\section{Após alguns dias de}

trabalho externo, a

equipe de vacinação

retornou novamente

para a UAPS,

dando continuidade

à imunização

contra influenza,

juntamente com

as outras vacinas

do calendário

de imunização

já previstas $\mathrm{e}$

consideradas

\section{de rotina.}

\section{RESULTADOS E DISCUSSÃO}

Diante da situação de emergência em saúde, instalada com o advento do novo coronavírus, foi lançado o Plano de Contingência Municipal da Secretaria Municipal de Saúde de Fortaleza-SMS, elaborado em março de 2020, sob o intuito de nortear as estratégias de atuação junto à população, a partir de sua realidade, nos três níveis de atenção (primária, secundária e terciária). Tal documento passou por sucessivas atualizações, sendo a última datada de 5 de outubro de 2020 (versão 14.0) ${ }^{9}$.

Nesse Plano, no tocante à imunização, a SMS de Fortaleza orientou que na Atenção Primária à Saúde-APS não deveria, de forma alguma, suspender o atendimento da vacinação ${ }^{10}$. Na UAPS objeto do presente estudo, os profissionais acordaram, junto com a coordenação, numa estratégia específica para esse atendimento, através de fluxos diferenciados de acordo com as necessidades dos usuários: por uma entrada da UAPS se dava o acolhimento das pessoas com síndromes gripais, e por outro acesso ocorria a assistência de usuários não sintomáticos, dentre eles, a população em busca de vacinação.

A antecipação da campanha ocorreu por decisão do Ministério da Saúde, considerando o momento histórico de combate ao coronavírus em escala global, e em especial pela confirmação de dois casos no país ainda no primeiro trimestre do ano. A vacina contra influenza não previne contra o novo vírus, mas com a imunização protege-se a população das doenças originadas pelo vírus da influenza, o que minimiza o impacto sobre os serviços de saúde. Outra questão importante a favor da imunização é que a sintomatologia das duas patologias têm semelhanças, e, com a antecipação, procurou-se reduzir a carga da circulação de influenza no meio populacional ${ }^{3}$.

Para essa ação, foi realizada mobilização por parte dos ACS dentro da comunidade, elaborados e impressos mini folhetos educativos com orientações sobre os cuidados com o novo coronavírus, onde a população poderia procurar ajuda e telefones de locais ligados à saúde pública para as testagens sobre diagnóstico do COVID $-19^{9}$.

A Residência Multiprofissional proporciona um olhar biopsicossocial do profissional residente sobre as demandas e os adoecimentos que os cercam. Isso vinculado ao caráter interdisciplinar do cui- 


\section{artigo}

dado, fortalece saberes e vínculos que são transformados em ações extra-ambulatoriais, reduzindo fragilidades do serviço, e criando iniciativas que beneficiam pacientes, profissionais, e as próprias unidades de saúde, como essa interação com a Campanha de Vacina contra a Influenza ${ }^{10}$.

$\mathrm{O}$ esquema de vacinação seguiu um fluxo o qual era dividido entre dois espaços de atuação: os profissionais da sala de vacina, dentro da UAPS, recebiam os usuários que precisavam atualizar sua caderneta de vacinação com o que era previsto de acordo com o Calendário Nacional de Imunização, enquanto havia outra equipe dentro da igreja do bairro que imunizava diariamente contra influenza.

Nesse período de estado latente da contaminação pelo novo coronavírus, a equipe pensou em metodologias apropriadas à realidade, vivenciado esse momento de forma coletiva, imunizando para além dos usuários pertencentes à área de abrangência, pois todos os dias, diversas pessoas se deslocavam de suas áreas mais distantes para a UAPS aqui relatada, à procura de atendimento, sendo por sua vez, todos atendidos.

Nesse aspecto, as atividades de educação em saúde extrapolam o domínio técnico-científico de cada profissão e estendem-se a prática de relações em todos os componentes de relevância e interesse social, contribuindo para a elevação da qualidade de saúde da população, enfrentamento de aspectos epidemiológicos do processo saúde-doença e na organização da estrutura do cuidado à saúde e gestão setorial ${ }^{11}$.

\section{CONCLUSÃO}

Trabalho Multiprofissional com envolvimento biopsicossocial possibilita o desenvolvimento de atividades, indepen- dente da atividade profissional específica de cada área envolvida, pois há uma inter-relação de conhecimentos para um acompanhamento diferenciado, possibilitando atendimento humanizado.

Atividades de educação em saúde desenvolvidas por uma equipe multidisciplinar expande os horizontes para o desenvolvimento de atividades de maneira integral, apresentando-se como uma estratégia promissora no enfrentamento aos diversos problemas de saúde que afetam a população.

A adoção das formas educativas relacionadas neste trabalho mostrou que apesar de um contexto novo de pandemia, as atividades realizadas uniram conhecimento técnico e prático em uma dada realidade de saúde, compartilhando-se informações importantes entre usuários, familiares e profissionais, o que refletiu no cuidado de saúde das pessoas e da comunidade.

\section{REFERÊNCIAS}

1. Brasil. Ministério da Saúde. Portal da Saúde. Sistema de informação de agravos de notificação - SINAN. Monitoramento dos casos de influenza no Brasil, Semanas Epidemiológicas 1 a 32 de 2019 [Internet]. 2019 [citado em 24 out 2020] Disponível em: https://antigo.saude.gov.br/images/pdf/2019/setembro/06/ BE-21-influenza-04set19.pdf

2. Silva MRV et al. Imunização: O conhecimento e práticas dos profissionais de enfermagem na sala de vacina. Revista Nursing. 2020 [citado em 25 out 2020] 23(260):3533 - 3536. Disponivel em: https://pesquisa.bvsalud.org/portal/resource/pt/ biblio-1095551

3. Brasil. Ministério da Saúde. Secretaria de Vigilância em Saúde. Departamento de Vigilância das Doenças Transmissíveis.Influenza: Monitoramento até a Semana Epidemiológica 49 de 2019 [recurso eletrônico] / Ministério da Saúde, Secretaria de Vigilância em Saúde, Departamento de Vigilância das Doenças Transmissíveis. - Brasília : Ministério da Saúde, 2019 [citado em 26 out 2020] 43 p. Disponivel em: https://antigo.saude.gov. br/images/pdf/2019/dezembro/23/Boletim-epidemiologico-SVS-38-2-interativo.pdf

4. Brasil. Ministério da Saúde. Informe Técnico. 22a Campanha Nacional de Vacinação contra a Influenza. [recurso eletrônico] / Ministério da Saúde, Secretaria de Vigilância em Saúde, Departamento de Vigilância das Doenças Transmissíveis. - Brasília : Ministério da Saúde, 2020 [citado em 26 out 2020] 30 p. Disponivel em: https://sbim.org.br/images/files/notas-tecnicas/informe-tecnico-ms-campanha-influenza-2020-final.pdf

5. Falkenbergn $\mathrm{MB}$ et al. Educação em saúde e educação na saúde: conceitos e implicações para a saúde coletiva. Ciência \& Saúde Coletiva. 2014 [citado em 26 out 2020] 19(3): 847-852.

6. Gil AC, editor. Como elaborar projetos de pesquisa. 4. ed. São Paulo, Brasil: Atlas; 2008.

7. Velho MB et al. Arco de Charles Maguerez: refletindo estratégias de metodologia ativa na formação de profissionais de saúde. Esc. Anna Nery Rev. Enferm, 2012 [citado em 27 out 2020] 16(1):172-177. Disponível em: https://www.scielo.br/scielo.php?script=sci_arttext\&pid=S1414-81452012000100023

8. Ventura MM. O estudo de caso como modalidade de pesquisa. Ver. SOCERJ. 2012. 20(5):383-386, set/out.

9. Fortaleza. Secretaria Municipal de Saúde. Plano Municipal de Contingência para enfrentamento da Infecção Humana pelo Novo Coronavírus SARS-CoV-2, Versão 14.0, Atualização 05/10/2020. Modo de acesso: World Wide Web: https://saude. fortaleza.ce.gov.br/images/coronavirus/PDFS/Plano-de-contingencia.pdf

10. Rebouças HNR et al. Residência Multiprofissional: Contribuições Durante a Pandemia.Cadernos Esp. Ceará-Edição Especial. 2014 [citado em 28 out 2020 14(1):128-132, jan.jun. Disponível em: file://C:/Users/w10c/Downloads/365-Article\%20Text-1671-1-10-20200722.pdf

11. Ceccim RB, Feuerwerker LCM. O Quadrilátero da Formação para a Área da Saúde: Ensino, Gestão, Atenção e Controle Social. Revista de Saúde Coletiva 2004 [citado em 28 out 2020] .Rio de Janeiro; 14(1):41-65. Disponivel em: file:///C:/Users/w10c/ Downloads/365-Article\%20Text-1671-1-10-20200722.pdf 